\title{
Benefits of a Molecular-Based Method for the Detection of Clarithromycin-Resistant Helicobacter pylori
}

\author{
Batsaikhan Saruuljavkhlan ${ }^{1}$ and Yoshio Yamaoka ${ }^{1,2}$ \\ ${ }^{1}$ Department of Environmental and Preventive Medicine, Faculty of Medicine, Oita University, Yufu, Japan, and ${ }^{2}$ Section of \\ Gastroenterology and Hepatology, Department of Medicine, Baylor College of Medicine, Houston, TX, USA
}

Corresponding Author

Yoshio Yamaoka

ORCID https://orcid.org/0000-0002-1222-5819

E-mail yyamaoka@oita-u.ac.jp
See "Types of 23S Ribosomal RNA Point Mutations and Therapeutic Outcomes for Helicobacter pylori” by Sang Yoon Kim, et al. on page 528, Vol. 15, No. 4, 2021
Gastric cancer is estimated to be the fifth most common cancer and the fourth leading cause of cancer deaths worldwide. ${ }^{1}$ The occurrence of gastric cancer is linked to commonly-occurring Helicobacter pylori infection. Because of difficulties in developing a vaccine, $H$. pylori eradication treatment has emerged as a key strategy for limiting infection and preventing complications, such as precancerous diseases, in the general population. ${ }^{2}$ Antibiotic resistance has increased, albeit with regional differences in rates of resistance, and affects first-line and other rescue treatments. A survey of 16 countries in the Asian-Pacific region found that resistance rates to clarithromycin in 11 countries and to levofloxacin in 13 countries were greater than $15 \%{ }^{3}$ To address this, improved detection methods and novel treatment strategies based on a deeper understanding of bacterial resistance mechanisms are needed.

In this issue of Gut and Liver, Kim et al. ${ }^{4}$ examined the prevalence of resistance to clarithromycin related to point mutations using a dual-priming oligonucleotide (DPO)based multiplex polymerase chain reaction (PCR) assay and assessed the impact of different point mutations on the success of eradication therapy. This retrospective cohort study examined 464 patients by reviewing databases in Korea between June 2014 and October 2019. The authors found that standard triple therapy was effective in more cases in the negative point mutation group compared with the positive point mutation group $(\mathrm{p}<0.001)$. The point mutation rate was $37.7 \%$ among the study population, with the single mutation $\mathrm{A} 2143 \mathrm{G}$ being most frequently identified, followed by A2142G, and double point mutations.
In 2017, Kuo et al. ${ }^{3}$ reported that the mean prevalence of resistance to clarithromycin was $17 \%$ in Korea. This indicated that the DPO-based multiplex PCR assay was effective at detecting cases that had gained clarithromycin resistance with minor conformational changes. The authors analyzed the cure rate of standard triple versus bismuthquadruple therapy in the negative, A2142G, and A2143G or double point mutation groups, and found rates of $89.8 \%$ and $100.0 \% ; 100.0 \%$ and $100.0 \%$; and $25.8 \%$ and $92.1 \%$ by per-protocol analysis, respectively. Standard triple therapy therefore had a similar cure rate to bismuth-quadruple therapy in the A2142G mutation group. According to research from various countries, both the A2142C/G and A2143G mutations are common with or without other mutations. A2143G induces a wide range of minimum inhibitory concentrations (MICs) (2-256 mg/L), whereas A2142 has a more restricted MIC of $64 \mathrm{mg} / \mathrm{L}$, which may make it responsive to treatment. ${ }^{5}$ However, because the study was limited to one center in Korea, further research is required to confirm these findings A2143G, but not A2142, may be associated with amoxicillin resistance as a synergistic effect. ${ }^{6}$ The identification of mutations can inform us on whether to avoid standard-line treatment or continue standard-line treatment at high dosages (or for a longer period). This study showed that 14-day bismuthquadruple therapy resulted in additional eradication success compared with 7 -day therapy $(\mathrm{p}=0.024)$. Although the study did not evaluate resistance to amoxicillin or other antibiotics, bismuth therapy showed higher efficiency in the A2143G and double point mutation groups. Generally, 
this study revealed the effectiveness of tailored therapy.

Despite the authors' excellent study design, there were some significant limitations. First, it is the disadvantages of a DPO-PCR method. The H. pylori resistance mechanism for clarithromycin is relatively well-known and mainly associated with $23 S$ rRNA gene (V domain) point mutations. ${ }^{7}$ This knowledge enables the development of several molecular-based methods. The DPO-PCR assay can only examine a limited number of nucleotide positions and is unable to determine new point mutations, with potential false negative results. ${ }^{7}$ Next generation sequencing techniques, such as MinION (Oxford Nanopore Technology), may provide a more complete picture of old and new resistance substitutions, as well as greater accuracy in detecting genome variations (heterozygote phenotypes) because they include two copies of the $23 S$ rRNA gene. ${ }^{7}$ Second, the analysis of failed cases was lacking. The authors did not exclude any potential causes of failure. Failed cases may be explained by other resistance mechanisms ${ }^{8}$ such as other point mutations in $\mathrm{rpl} 22$ or infB, an efflux pump system, or coccoid or biofilm formation, as shown in Fig. 1. ${ }^{7}$ However, how these mechanisms may affect clarithromycin resistance and their clinical relevance remain unclear, but it may be that different mechanisms act synergistically. ${ }^{7}$ Some studies have shown that isolates with $23 S$ rRNA mutations have 4-fold decreased MICs in the presence of efflux pump inhibitors. Besides the $23 S$ rRNA (V domain)related mechanism, other mutations may induce low-level clarithromycin resistance, and this resistance may be increased by the cooperative effects of A2142 and A2143 mutations. ${ }^{9}$ In cases of mutations in outside this domain, high doses of clarithromycin need to be used without exposure to any unnecessary antibiotics. Moreover, the spread of multidrug resistance ( $40 \%$ of infections) and host factors, including proton pump inhibitor activity due to CYP2C19 gene polymorphisms, can also reduce eradication success. Third, in this retrospective study, it would be more instructive to identify relationships between phenotype and genotype related to primary and secondary drug resistance.

Limitations notwithstanding, the importance of molecular-based methods for analyzing $H$. pylori resistance and understanding first-line therapy failure were revealed in this study. However, in the absence of a universal detection method for clarithromycin resistance, several methods have been introduced and the efficiency, safety, costeffectiveness, and availability of these methods should be evaluated and compared in multicenter studies. Regarding clinical usage, we should clarify other strategies of clarithromycin-based therapy (duration, doses, or combination with other drugs) in cases with low MIC isolates due

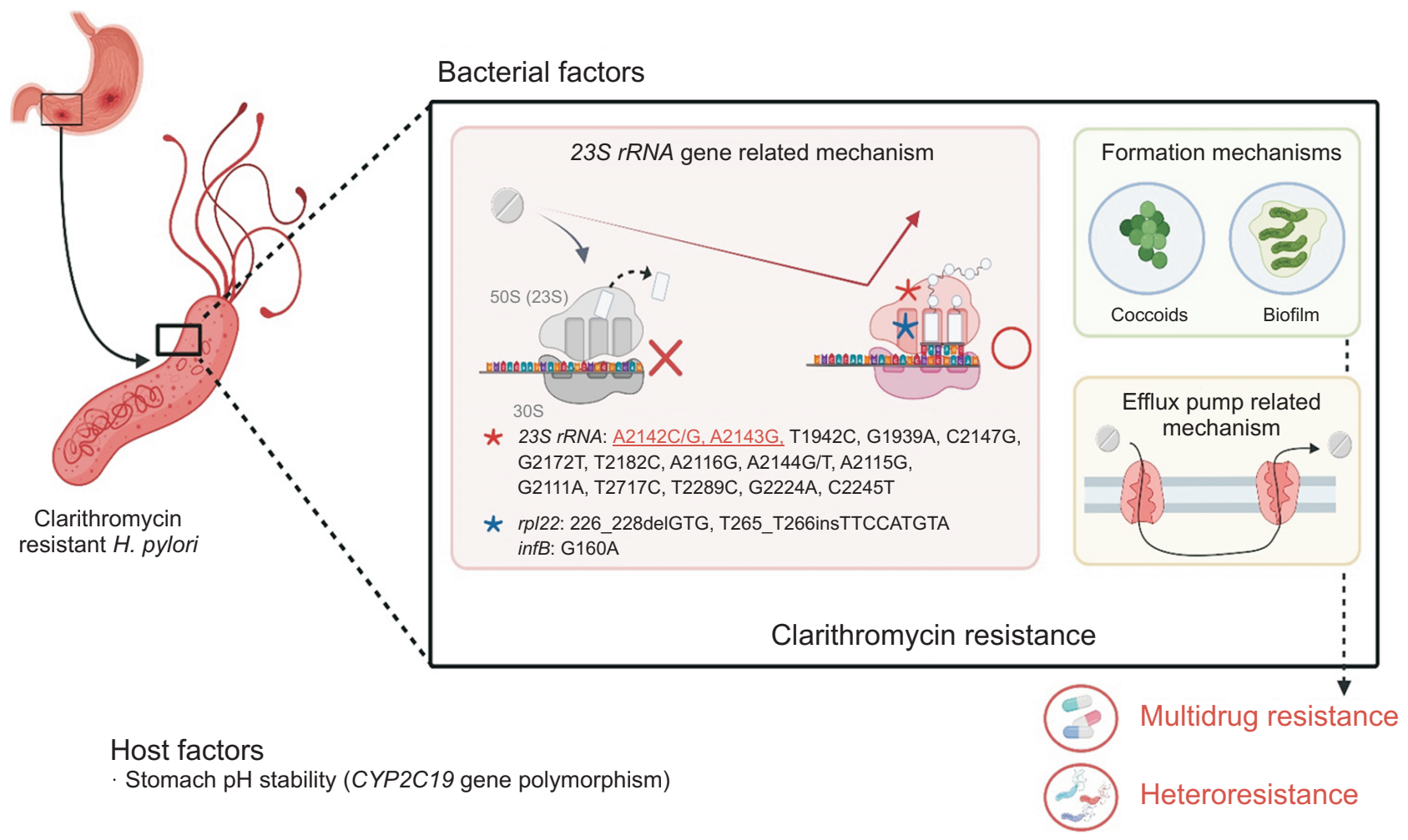

Fig. 1. Schematic explanation of clarithromycin resistance mechanisms in Helicobacter pylori infection. Generally, resistance of $H$. pylori to clarithromycin is mediated by three main mechanisms: targeted modification by genetic mutations such as in the $23 S$ rRNA gene; drug efflux systems; and other mechanisms such as biofilm or coccoid formation. 
to single or exclusive A2142 and A2143 point mutations.

In summary, Kim et al. ${ }^{4}$ studied how application of the DPO-based multiplex PCR assay in clinical practice to detect clarithromycin-resistant cases, may improve the cure rate of $H$. pylori infection. Future studies focused on tailored therapy should aim to reduce the incidence of resistance in $H$. pylori infections, apply this to clinical practice, and develop strategies that depend on population-specific resistance mechanisms. To this end, it may be important to consider diagnosing resistant $H$. pylori infections as a first step in countries with high prevalence of antibiotic resistance, i.e., "test with resistance check, treat and confirm eradication."

\section{CONFLICTS OF INTEREST}

No potential conflict of interest relevant to this article was reported.

\section{ACKNOWLEDGEMENTS}

This report is based on work supported in part by grants from Grants-in-Aid for Scientific Research from the Ministry of Education, Culture, Sports, Science, and Technology (MEXT) of Japan (221S0002, 16H06279, 18KK0266, 19H03473) (Y.Y.). B.S. is Ph.D. student supported by the MEXT scholarship program for 2019.

\section{ORCID}

Batsaikhan Saruuljavkhlan https://orcid.org/0000-0003-3258-4453

Yoshio Yamaoka https://orcid.org/0000-0002-1222-5819

\section{REFERENCES}

1. Sung H, Ferlay J, Siegel RL, et al. Global cancer statistics 2020: GLOBOCAN estimates of incidence and mortality worldwide for 36 cancers in 185 countries. CA Cancer J Clin 2021;71:209-249.

2. Yoon SB, Park JM, Lim CH, Cho YK, Choi MG. Effect of Helicobacter pylori eradication on metachronous gastric cancer after endoscopic resection of gastric tumors: a metaanalysis. Helicobacter 2014;19:243-248.

3. Kuo YT, Liou JM, El-Omar EM, et al. Primary antibiotic resistance in Helicobacter pylori in the Asia-Pacific region: a systematic review and meta-analysis. Lancet Gastroenterol Hepatol 2017;2:707-715.

4. Kim SY, Park JM, Lim CH, et al. Types of 23 S ribosomal RNA point mutations and therapeutic outcomes for Helicobacter pylori. Gut Liver 2021;15:528-536.

5. Marques AT, Vítor JMB, Santos A, Oleastro M, Vale FF. Trends in Helicobacter pylori resistance to clarithromycin: from phenotypic to genomic approaches. Microb Genom 2020;6:e000344.

6. Sakinc T, Baars B, Wüppenhorst N, Kist M, Huebner J, Opferkuch W. Influence of a $23 \mathrm{~S}$ ribosomal RNA mutation in Helicobacter pylori strains on the in vitro synergistic effect of clarithromycin and amoxicillin. BMC Res Notes 2012;5:603.

7. Tshibangu-Kabamba E, Yamaoka Y. Helicobacter pylori infection and antibiotic resistance: from biology to clinical implications. Nat Rev Gastroenterol Hepatol. Epub 2021 May 17. https://doi.org/10.1038/s41575-021-00449-x.

8. Ong S, Kim SE, Kim JH, et al. Helicobacter pylori eradication rates with concomitant and tailored therapy based on $23 \mathrm{~S}$ rRNA point mutation: a multicenter randomized controlled trial. Helicobacter 2019;24:e12654.

9. Binh TT, Shiota S, Suzuki R, et al. Discovery of novel mutations for clarithromycin resistance in Helicobacter pylori by using next-generation sequencing. J Antimicrob Chemother 2014;69:1796-1803. 